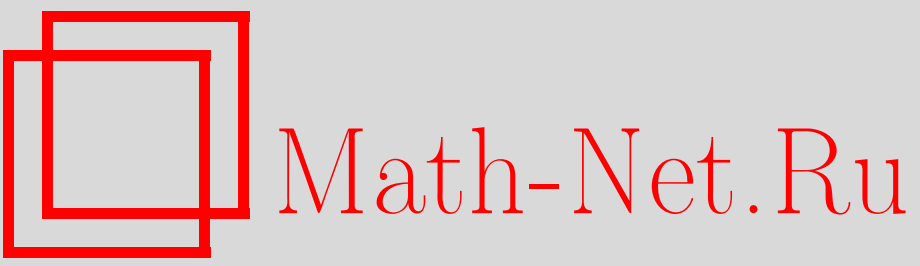

Э. Л. Пресман, И. М. Сонин, Об одной задаче оптимальной остановки для случайных величин, заданных на цепи Маркова, Теория вероятн. и ее примен., 2009, том 54, выпуск 3, 599-608

DOI: https://doi.org/10.4213/tvp2814

Использование Общероссийского математического портала Math-Net.Ru подразумевает, что вы прочитали и согласны с пользовательским соглашением

http://www . mathnet.ru/rus/agreement

Параметры загрузки:

IP : 54.196 .121 .252

26 апреля 2023 г., 11:52:26 


\title{
ОБ ОДНОЙ ЗАДАЧЕ ОПТИМАЛЬНОЙ ОСТАНОВКИ ДЛЯ СЛУЧАЙНЫХ ВЕЛИЧИН, ЗАДАННЫХ НА ЦЕПИ МАРКОВА ${ }^{1)}$
}

\begin{abstract}
Настоящая работа преследует две цели: во-первых, описание нового класса задач оптимальной остановки, для которых решение может быть либо найдено в явном виде, либо получено за конечное число шагов, а вовторых, обобщение на изучаемую ситуацию и демонстрация применения алгоритма исключения состояний (state elimination algorithm), pазработанного ранее одним из авторов для задачи оптимальной остановки конечной или счетной марковской цепи.
\end{abstract}

Ключевые слова и фразыл: цепи Маркова, оптимальная остановка, алгоритм исключения состояний, сезонные наблюдения.

1. Введение и формулировка результата. Рассмотрим три задачи, имеющие общую структуру, при этом каждая последующая является обобщением предыдущей. В каждой задаче статистик наблюдает последовательность $Z=\left(Z_{n}\right)_{n} \geqslant 0$ случайных величин. Цель - максимизировать $\mathbf{E} \beta^{\tau} g\left(Z_{\tau}\right)$ по всем моментам остановки $\tau$, где $\beta$ - коэффициент дисконтирования, $0<\beta \leqslant 1$, а функция $g$ определяет доход от остановки.

3 а д а ч а 1: классическая задача оптимальной остановки независимых испытаний (см., например, [6]). Наблюдается последовательность независимых одинаково распределенных случайных величин (н.о.р.с.в.) $\left(Z_{n}\right)_{n \geqslant 0}$ с известным распределением $F$ и $\beta<1$ (примером может служить последовательное подбрасывание шестигранной кости). Известно, что в этой задаче существует такое число $c^{*}=c^{*}(\beta)$, что оптимальным является момент первого попадания в множество $\left\{z: g(z) \geqslant c^{*}\right\}$.

3 а д а ч а 2: «сезонные наблюдения». Примером является ситуация, когда имеется $m$ различных неправильных костей, которые подбрасываются последовательно: первая, вторая, $\ldots, m$-я и опять - первая, вторая и т.д. Формально: заданы $m$ независимых последовательностей $\left(Y_{n}^{s}\right)_{n \geqslant 0}$ н.о.р.с.в. с известными распределениями $F(s, \cdot)$, $s \in B=\{1,2, \ldots, m\}, \beta<1$. Последовательность наблюдений $\left(Z_{n}\right)_{n \geqslant 0}$ имеет вид $Z_{n}=Y_{n}^{s}$, где $s \equiv n(\bmod m), s \in B$. Задача 1 получается при $m=1$.

3 а д а ч а 3: «общие сезонные наблюдения». Примером является ситуация, когда состояние марковской цепи со значениями в множестве $B$ определяет в каждый момент времени ту из неправильных костей, которая подбрасывается в этот момент. Как и ранее, заданы $m$ независимых последовательностей $\left(Y_{n}^{s}\right)_{n} \geqslant 0$ н.о.р.с.в. с известными распределениями $F(s, \cdot), s \in B$, и, кроме того, не зависящая от них марковская цепь $\left(\widetilde{U}_{n}\right)_{n \geqslant 0}$, принимающая значения в $B$ с заданными переходными вероятностями $\widetilde{p}_{s, k}$, и задано $\beta<1$. Последовательность наблюдений $\left(\widetilde{Z}_{n}\right)_{n \geqslant 0}$ имеет вид $\widetilde{Z}_{n}=\left(\widetilde{U}_{n}, Y_{n}^{\widetilde{U}_{n}}\right)$. Задача 2 получается, когда $\left(\widetilde{U}_{n}\right)_{n \geqslant 0}$ соответствует детерминированному циклическому движению по множеству $B$.

Напомним, что случай $\beta<1$ сводится к случаю $\beta=1$ введением поглощающего состояния. В рассматриваемом случае можно ввести поглощающее состояние

* Центральный экономико-математический институт РАН, Нахимовский проспект, 47, 117428 Москва, Россия; e-mail: presman@cemi.rssi.ru

** Dept. of Mathematics, Univ. of North Carolina at Charlotte, Charlotte, NC 28223, USA; ЦЭМИ РАН, Москва, Россия; е-mail: imsonin@uncc.edu

1) Работа первого автора выполнена при поддержке РФФИ (проект 07-01-00541). 
$e=(\widetilde{e}, 0)$, вместо $\left(\widetilde{U}_{n}\right)_{n} \geqslant 0$ рассмотреть новую цепь $\left(U_{n}\right)_{n \geqslant 0}$ со значениями в $\widetilde{e} \cup B$ и переходными вероятностями $p_{s, k}=\beta \widetilde{p}_{s, k}, s, k \in B ; p_{s, \tilde{e}}=1-\beta, s \in B$, и положить $Y_{n}^{\tilde{e}} \equiv 0, g(e)=0$. Тогда для любого момента остановки $\tau$

$$
\mathbf{E}\left[\beta^{\tau} g\left(\widetilde{U}_{\tau}, Y_{\tau}^{\widetilde{U}_{\tau}}\right)\right]=\mathbf{E}\left[g\left(U_{\tau}, Y_{\tau}^{U_{\tau}}\right)\right] .
$$

В работе рассматривается несколько более общая постановка, чем задача 3 , когда вероятность перехода в поглощающее состояние может зависеть от состояния марковской цепи $\left(\widetilde{U}_{n}\right)_{n \geqslant 0}$. Мы считаем, что на вероятностном пространстве $(\Omega, \mathscr{F}, \mathbf{P})$ задана такая последовательность случайных величин $Z=\left(Z_{n}\right)_{n \geqslant 0}$ со значениями в $X=\{e\} \cup(B \otimes \mathbf{R})$, что $Z_{n}=\left(U_{n}, Y_{n}\right), n \geqslant 0$, и

$$
\begin{aligned}
& \mathbf{P}\left[Z_{n+1}=e \mid Z_{0}, \ldots, Z_{n-1}, Z_{n}=e\right]=1, \\
& \mathbf{P}\left[U_{n+1}=j, Y_{n+1} \in A \mid Z_{0}, \ldots, Z_{n-1}, Z_{n}=z=(i, y)\right]=p_{i j} F(j, A), \\
& \mathbf{P}\left[Z_{n+1}=e \mid Z_{0}, \ldots, Z_{n-1}, Z_{n}=z=(i, y)\right]=1-\sum_{j \in B} p_{i j}
\end{aligned}
$$

для $i, j \in B, y \in \mathbf{R}$, где $F(j, \cdot)$ - некоторые вероятностные меры на борелевской прямой $\mathbf{R}, F(j, \mathbf{R})=1, j \in B$, а $P=\left(p_{i j}, i, j \in B\right)$ - строго субстохастическая матрица, т.е. такая матрица с неотрицательными элементами, что

$$
\sum_{j \in B} p_{i j}<1 \text { для всех } i \in B .
$$

Очевидно, что последовательности $\left(Z_{n}\right)_{n \geqslant 0}$ и $\left(U_{n}\right)_{n \geqslant 0}$ образуют цепи Маркова, при этом цепь $\left(U_{n}\right)_{n \geqslant 0}$, переходные вероятности которой вне поглощающего состояния определяются матрицей $P$, является в некотором смысле основной цепью, которая «управляет» последовательностью $\left(Y_{n}\right)_{n} \geqslant 0$. В этой ситуации обычно говорят о последовательности случайных величин $\left(Y_{n}\right)_{n \geqslant 0}$, заданньх на чепи Маркова $\left(U_{n}\right)_{n \geqslant 0}$, или что чепь $Z$ определяется матрицей $P$ и мерами $F(j, \cdot), j \in B$. Если $\sum_{j \in B} p_{i j}=\beta$ для всех $i \in B, 0<\beta<1$, то получаем задачу, эквивалентную задаче 3 , с переходными вероятностями $\widetilde{p}_{i j}=p_{i j} / \beta, i, j \in B$. Отметим, что условие (2) можно ослабить, считая, например, что этому условию удовлетворяет не сама матрица $P$, а некоторая ее степень.

Мы считаем, что на $X$ задана такая измеримая функция $g(\cdot)$, что $g(e)=0$, $\int_{\mathbf{R}}|g(i, v)| F(i, d v)<\infty$ для всех $i \in B$, и рассматриваем задачу оптимальной остановки цепи $Z$, определяемой матрицей $P$ и мерами $F(i, \cdot)$, с функцией платы $g(\cdot)$ и $\beta=1$. Функцию выигрыша в этой задаче обозначим $V(z)=\sup _{\tau} \mathbf{E}\left[g\left(Z_{\tau}\right) \mid Z_{0}=z\right]$.

Основным результатом данной работы является приводимая ниже теорема, которая дает описание множества остановки и функции выигрыша для рассматриваемой задачи. Прежде чем формулировать теорему, введем следующие обозначения. Для любого $A=\left\{(j, y): j \in B, y \in A_{j}\right\}$, где $A_{j}, j \in B$, - некоторые (возможно пустые) множества из $\mathbf{R}$, обозначим через $F_{d}(A)$ диагональную матрицу

$$
F_{d}(A)=\left(\delta_{i j} F\left(j, A_{j}\right), i, j \in B\right) .
$$

Теорема. Существует такой вектор $d^{*}=\left(d_{1}^{*}, \ldots, d_{m}^{*}\right)$, ито

а) оптимальный момент остановки $\tau^{*}$ - это момент первого попадания цепи $Z$ в множество $\{e\} \cup D^{*}$, где $D^{*}=\left\{z=(i, y): i \in B, y \in D_{i}^{*}\right\}, D_{i}^{*}=\left\{y: g(i, y) \geqslant d_{i}^{*}\right\}$;

б) функция выигрыша удовлетворяет соотношению

$$
V(z)=g(z), \quad z \in D^{*}, \quad V(i, y)=d_{i}^{*}>g(i, y), \quad z=(i, y) \notin D^{*},
$$

и $d^{*}$ удовлетворяет соотночению

$$
d_{i}^{*}=\sum_{j \in B} p_{i j}^{*} \int_{D_{j}^{*}} g(j, v) F(j, d v)
$$


где матрица $P^{*}=\left(p_{i j}^{*}, i, j \in B\right)$ определяется равенством

$$
P^{*}=\left[I+P F_{d}\left(D^{*}\right)-P\right]^{-1} P
$$

u при фиксированном начальном состоянии $i$ величина $F\left(j, D_{j}^{*}\right) p_{i j}^{*}=\mathbf{P}\left[U_{\tilde{\tau}}=j \mid U_{0}=\right.$ i] определяет распределение первой координаты в момент $\widetilde{\tau}$ первого после нуля попадания чепи $Z$ в множество $D^{*}$;

в) существует алгоритм нахождения вектора $d^{*}$, а значит, и построения функиии выигрыша и множества остановки, который в случае дискретных распределений с носителями, не имеюшими конечньх предельных точек, приводит $к$ построению множества остановки и функиии выцгрыша за конечное число шагов.

Доказательство теоремы приводится в разделе 3. Там мы не только доказываем теорему, но и приводим упомянутый в пункте в) алгоритм (см. (22)-(24) и лемму 3). Алгоритм основан на общем алгоритме исключения состояний в задаче оптимальной остановки. Соответственно, в разделе 2 мы напомним основные факты из теории оптимальной остановки и обсудим общий алгоритм. Предварительные результаты данной работы для дискретного случая были сформулированы в [12].

2. Задача оптимальной остановки и алгоритм исключения состояний. Существуют два подхода к изучению задачи оптимальной остановки - мартингальный и марковский. Оба подхода интенсивно развивались в середине шестидесятых годов. Мартингальный подход был изложен в книге Г.Роббинса, Д. Сигмунда и И. Чао (1971) [3] (изложение этого подхода можно найти также в книге Т. С. Фергюсона [6]). Марковский подход был изложен в книгах А. Н. Ширяева (1969, 1976) [4] и Е. Б. Дынкина и А. А. Юшкевича (1969) [1]. Из многочисленных книг и монографий с главами или разделами, посвященными задаче оптимальной остановки, упомянем [5] и [8]. Современное изложение обоих подходов приведено в монографии Г. Пешкира и А. Н. Ширяева [7]. Наше изложение использует марковский подход.

Пусть на измеримом пространстве $(\Omega, \mathscr{F})$ заданы такая последовательность случайных элементов $\left(Z_{n}\right)_{n \geqslant 0}$ со значениями в фазовом пространстве $(X, \mathscr{B})$ и такая совокупность мер $\mathbf{P}_{z}$, измеримым образом зависящая от $z \in X$, что $Z=\left(Z_{n}, \mathscr{F}_{n}, \mathbf{P}_{z}\right)_{n \geqslant 0}$ образует однородную марковскую цепь, где $\mathbf{P}_{z}\left[Z_{0}=z\right]=1$, а $\mathscr{F}_{n}$ обозначает $\sigma$ алгебру, порожденную величинами $Z_{0}, \ldots, Z_{n}$. Заметим, что в этом разделе цепь $Z$ носит общий характер и не обязана совпадать с цепью из предыдущего раздела. Обозначим через $\mathscr{P}$ переходной оператор цепи $Z$, так что $\mathscr{P} f(z)=\mathbf{E}_{z}\left[f\left(Z_{1}\right)\right]$. Оператор $\mathscr{P}$ переводит любую заданную на $X$ измеримую функции $f$, для которой соответствующее математическое ожидание определено, в измеримую функцию, заданную на $X$.

Считаются заданными число $\beta, 0<\beta \leqslant 1$, и измеримые функции $g(z)$ и $c(z)$. Моменты остановки рассматриваются относительно последовательности $\sigma$-алгебр $\mathscr{F}_{n}$, $n \geqslant 0$. Предполагается, что для любого $z \in X$ и любого момента остановки $\tau$ определена величина $V_{\tau}(z)=\mathbf{E}_{z}\left[g\left(Z_{\tau}\right) \beta^{\tau}-\sum_{k=0}^{\tau-1} c\left(Z_{k}\right) \beta^{k}\right]$. Здесь $g(z)-$ это доход от остановки в точке $z$, а $c(z)$ — это плата за проведение очередного наблюдения (обе функции могут принимать как положительные, так и отрицательные значения). Задача оптимальной остановки состоит в нахождении функции выигрыша

$$
V(z)=\sup _{\tau} V_{\tau}(z)=\sup _{\tau} \mathbf{E}_{z}\left[g\left(Z_{\tau}\right) \beta^{\tau}-\sum_{k=0}^{\tau-1} c\left(Z_{k}\right) \beta^{k}\right],
$$

где супремум берется по всем моментам остановки, и нахождении оптимального момента остановки, т.е. момента, на котором достигается этот супремум, если такой момент существует. Как и в предыдущем разделе, случай $0<\beta<1$ сводится к случаю $\beta=1$ введением поглощающего состояния. Поэтому в дальнейшем без ограничения общности мы будем считать, что $\beta=1$. 
Определим оператор $\mathscr{T}$ следующим образом:

$$
\mathscr{T} f(z)=-c(z)+\mathscr{P} f(z) .
$$

Оператор $\mathscr{T}$ называется оператором переоценки.

Хорошо известно, что при выполнении естественных условий функция $V(z)$ конечна и справедливо следующее утверждение (см. [7, теорема 1.11 , следствие 1.12 и раздел 11 гл. 1]).

Утверждение 1. а) Функиия $V(z)$ является минимальныл решением уравнения оптимальности (уравнения Беллмана)

$$
V(z)=\max [g(z), \mathscr{T} V(z)]
$$

б) Если $\mathbf{P}_{z}\left[\tau^{*}<\infty\right]=1$ для всех $z \in X$, где $\tau^{*}=\inf \left\{n \geqslant 0: Z_{n} \in D^{*}\right\}, D^{*}=$ $\{z: V(z)=g(z)\}$, то момент остановки $\tau^{*}$ является оптимальным $u \tau^{*} \leqslant \tau^{\prime} \mathbf{P}_{z}$-п.н. для любого $z$ и любого оптимального момента остановки $\tau^{\prime}$.

в) Для последовательности $\widetilde{V}^{(0)}(z)=g(z), \widetilde{V}^{(k+1)}(z)=\max \left[g(z), \mathscr{T} \widetilde{V}^{(k)}(z)\right]$ сnраведливо $\widetilde{V}^{(k)} \uparrow V$.

Множество $D^{*}$ называется множеством остановки, а множество $C^{*}=X \backslash D^{*}=$ $\{z: V(z)>g(z)\}-$ множеством продолжения.

Обычно говорят, что утверждение в) дает конструктивный метод вычисления функции $V(z)$. Заметим, однако, что, как правило, $\widetilde{V}^{(k+1)} \neq \widetilde{V}^{(k)} \neq V$ для всех $k$. Это справедливо, даже если $Z_{n}$ принимает только два значения $z_{1}$ и $z_{2}$, цепь связна и $g\left(z_{1}\right) \neq g\left(z_{2}\right)$. Если $Z_{n}$ принимает конечное число значений, то уравнение (9) можно решать, используя методы линейного программирования, но при таком подходе теряется вероятностный смысл решения задачи и непонятно, как обобщить такой подход хотя бы на счетный случай. В работах [9]-[11] для конечного, а в некоторых ситуациях для счетного случая был предложен алгоритм построения функции $V(z)$, который основан на исключении состояний и гарантирует, что если $Z_{n}$ принимает $m$ значений, $m<\infty$, то после не более чем $(m-1)$ шагов будет найдено $D^{*}$, а затем после того же числа шагов будет найдено $V(z)$ для всех $z$. Там же говорится, что иногда этот алгоритм позволяет находить $V(z)$ и $D^{*}$ за конечное число шагов и тогда, когда фазовое пространство является счетным.

Алгоритм основан на следующих соображениях (см. [10], [11]).

1. В задаче оптимальной остановки трудно найти множество, где нужно останавливаться, но сравнительно легко найти множество, где останавливаться заведомо не нужно. В самом деле, останавливаться нужно в таких точках $z$, что $g(z) \geqslant \mathscr{T} V(z)$, но функция $V$ неизвестна, если задача еще не решена. С другой стороны, заведомо не нужно останавливаться в тех точках $z$, для которых $g(z)<\mathscr{T} g(z)$, т.е. когда доход от остановки в данный момент меньше, чем ожидаемый доход от остановки в следующий момент.

2. После того, как найдено некоторое множество $C$ точек $z$, в которых заведомо не нужно останавливаться, можно рассматривать начальное состояние цепи только из дополнительного множества $D=X \backslash C$, а саму исходную цепь только в моменты попадания в это дополнительное множество, т.е. рассматривать вложенную марковскую цепь. При этом нужно найти переходной оператор новой цепи и сделать переоценку платы за испытания, рассматривая в качестве новой платы математическое ожидание суммы старых плат, взятой между моментами последовательных попаданий в дополнительное множество. Тем самым мы исключаем состояния, в которых заведомо не нужно останавливаться, ибо новая цепь принимает значения в множестве $D$. Почти очевидно (и ниже мы это доказываем), что множество остановки для новой цепи совпадает с множеством остановки для старой и что функция выигрыша для старой цепи совпадает с функцией выигрыша для новой на множестве $D$, а на исключенном множестве $C$ равна сумме математического ожидания от значений функции выигрыша 
в новой задаче в момент первого попадания в множество $D$ и суммарной платы за наблюдения до этого момента.

3. Может оказаться, что после конечного числа применений указанной процедуры мы придем к ситуации, когда для новой цепи при всех $z$ из оставшегося множества $\widetilde{D}$ будет выполняться неравенство

$$
g(z) \geqslant \widetilde{\mathscr{T}} g(z), \quad z \in \widetilde{D}
$$

где $\widetilde{c}(z)$ - плата за испытание, а $\widetilde{\mathscr{T}}$ - оператор переоценки новой цепи. Если $Z_{0} \in \widetilde{D}$, то, тем самым, соотношение (10) будет выполняться для всего фазового пространства новой цепи. Заметим, что если у исходной цепи фазовое пространство состоит из $m$ элементов, то указанная ситуация получится не позже, чем через $m-1$ шагов. Но из утверждения 1 вытекает, что если соотношение (10) выполняется для всего фазового пространства новой цепи, то множество остановки совпадает со всем фазовым пространством, а функция выигрыша совпадает с платой за остановку. Тем самым будет найдена функция выигрыша исходной цепи на новом фазовом пространстве и останется пересчитать ее для $z$ из множества продолжения.

В работе [10] приведена вычислительная процедура реализации указанного алгоритма для конечного числа состояний и указано на возможность обобщения на счетный случай, если после конечного числа итераций получится соотношение (10). В paботе [2] была предложена модификация этого алгоритма, позволяющая рассматривать произвольное пространство состояний. Эта модификация связана с рассмотрением новой цепи с произвольным начальным состоянием, которое может не принадлежать множеству, дополнительному к исключаемому согласно исходному алгоритму. Она основана на приводимых ниже лемме 1 и следствии к ней (см. [2]). Отметим, что вариант этой леммы, отвечающий сужению на множество $D$, был получен для счетного случая в [13]. Для простоты рассмотрим случай $c(z) \equiv 0$.

Для любого $A \subset X$ наряду с оператором $\mathscr{P}$ будем рассматривать также оператор $\mathscr{P}_{A}$, определяемый равенством

$$
\mathscr{P}_{A} f=\mathscr{P}_{A} f
$$

где $I_{A}$ - оператор умножения на характеристическую функцию множества $A$.

Пусть задано некоторое $D \subset X$. Обозначим через $C$ дополнение множества $D$, т.е. $C=X \backslash D$. В дальнейшем дополнение любого множества $D$ (с индексами и/или с указанием зависимости от параметров) будем обозначать через $C$ с теми же индексами и/или с указанием той же зависимости от параметров.

Пусть $\tau_{0}=0$, и пусть $\tau_{n}>0, n \geqslant 1,-$ моменты последовательного попадания цепи $Z$ в множество $D$ (если $Z_{0}=z$ и $z \in D$, то $\tau_{1}$ - это момент первого возвращения). Пусть известно, что $\mathbf{P}_{z}\left[\tau_{1}<\infty\right]=1$ для любого $z \in X$. Рассмотрим марковскую цепь $Z^{\prime}=\left(Z_{n}^{\prime}\right)_{n \geqslant 0}$, где $Z_{n}^{\prime}=Z_{\tau_{n}}, n \geqslant 0$. Обозначим через $\mathscr{P}^{\prime}$ переходной оператор цепи $Z^{\prime}$, а через $I$ тождественный оператор, т.е. $I=I_{X}$.

Лемма 1. Справедливы соотношения

$$
\begin{aligned}
& \mathscr{P}^{\prime} f=\sum_{l=0}^{\infty}\left(\mathscr{P}_{C}\right)^{l} \mathscr{P}_{D} f=\left(I-\mathscr{P}_{C}\right)^{-1} \mathscr{P}_{D} f, \\
& \mathscr{P}^{\prime} f=\mathscr{P}_{f}+\left(I-\mathscr{P}_{C}\right)^{-1} \mathscr{P}_{C}\left(\mathscr{P}_{f}-f\right) .
\end{aligned}
$$

Д о к а $з$ а т е л ь с т в о. Второе равенство в (12) вытекает из определения оператора $\left(I-\mathscr{P}_{C}\right)^{-1}$, а первое соответствует формуле полной вероятности по системе событий $\left\{\tau_{1}=l+1\right\}, l \geqslant 0$, поскольку $\left(\mathscr{P}_{C}\right)^{l} \mathscr{P}_{D} f(z)$ - это результат усреднения $f\left(Z_{l+1}\right)$ по траекториям, которые начинаются в точке $z$, затем $l$ моментов проводят в $C$, а затем переходят в $D$. 
Подставляя в правую часть (12) равенство $\mathscr{P}_{D}=\mathscr{P}-\mathscr{P}_{C}$, а затем используя равенство $\left(I-\mathscr{P}_{C}\right)^{-1}=I+\left(I-\mathscr{P}_{C}\right)^{-1} \mathscr{P}_{C}$, получаем

$$
\begin{aligned}
\mathscr{P}^{\prime} & =\left(I-\mathscr{P}_{C}\right)^{-1} \mathscr{P}-\left(I-\mathscr{P}_{C}\right)^{-1} \mathscr{P}_{C} \\
& =\left(I+\left(I-\mathscr{P}_{C}\right)^{-1} \mathscr{P}_{C}\right) \mathscr{P}-\left(I-\mathscr{P}_{C}\right)^{-1} \mathscr{P}_{C} \\
& =\mathscr{P}+\left(I-\mathscr{P}_{C}\right)^{-1} \mathscr{P}_{C} \mathscr{P}-\left(I-\mathscr{P}_{C}\right)^{-1} \mathscr{P}_{C},
\end{aligned}
$$

что эквивалентно (13). Это завершает доказательство леммы 1.

Заметим, что в случае конечного числа состояний оператору $\left(I-\mathscr{P}_{C}\right)^{-1}$ отвечает матрица, которая называется фундаментальной матрицей для матрицы, отвечающей $\mathscr{P}_{C}$. Элементы этой матрицы равны среднему числу посещений состояния до момента выхода из множества $C$.

Из (13) вытекает следующее утверждение.

Следствие 1. Если $C \subseteq\{z: \mathscr{P} f(z)>f(z)\}$, mо $\mathscr{P}^{\prime} f(z) \geqslant \mathscr{P} f(z)$ для любого $z \in X$.

В следующем разделе мы демонстрируем, как лемму 1 можно использовать для построения множества остановки и функции выигрыша для задачи оптимальной остановки, рассмотренной в разделе 1 .

3. Случайные величины, заданные на цепи Маркова. Пусть марковская цепь $Z$ определяется строго субстохастической матрицей $P$ и вероятностными мерами $F(i, \cdot), i \in B$, заданными на всей прямой. Рассмотрим переходной оператор $\mathscr{P}$ нашей цепи на множестве таких функций, что $f(e)=0$. Поскольку состояние $e$ поглощающее, то для описания переходного оператора достаточно рассмотреть его сужение на множество функций, заданных на $B \otimes \mathbf{R}$. Соответствующее сужение мы также обозначаем через $\mathscr{P}$. Функции, заданные на $B \otimes \mathbf{R}$, можно рассматривать как векторфункции $f(y)=(f(1, y), \ldots, f(m, y))$, заданные на $\mathbf{R}$. На множестве таких функций определим оператор $\mathscr{L}$ по формуле

$$
\mathscr{L} f(i, y)=\int_{\mathbf{R}} f(i, v) F(i, d v), \quad(i, y) \in B \otimes \mathbf{R} .
$$

Из определения оператора $\mathscr{L}$ вытекает, что он переводит любую вектор-функцию в вектор-функцию, координаты которой равны константам, а значит, этим же свойством обладает оператор $\mathscr{P}$ и

$$
\mathscr{P} f=P \mathscr{L} f .
$$

Поэтому в дальнейшем мы будем говорить, что марковская цепь $Z$ определяется строго субстохастической матрицей $P$ и оператором $\mathscr{L}$ в $(15)$.

Для любого $A=\left\{(j, y): j \in B, y \in A_{j}\right\}$ наряду с оператором $\mathscr{L}$ будем рассматривать также оператор $\mathscr{L}_{A}$, определяемый равенством

$$
\mathscr{L}_{A} f=\mathscr{L} I_{A} f .
$$

Отсюда и из (11) и (15) следует, что

$$
\mathscr{P}_{A} f=P \mathscr{L}_{A} f .
$$

Прежде чем доказывать теорему, сформулируем ряд вспомогательных утверждений. Пусть задано некоторое $D=\left\{(j, y): j \in B, y \in D_{j}\right\}$. Пусть $\tau_{0}=0$, и пусть $\tau_{n}>0, n \geqslant 1$, моменты последовательного попадания цепи $Z$ в множество $\{e\} \cup D$ (если $Z_{0}=z$ и $z \in\{e\} \cup D$, то $\tau_{1}$ - это момент первого возвращения). Очевидно, что $\tau_{1} \leqslant \tau_{e}$, где $\tau_{e}-$ момент первого попадания в состояние $e$. Так как $P$ строго субстохастична, то $\mathbf{P}_{z}\left[\tau_{e}<\infty\right]=1$, а значит, $\mathbf{P}_{z}\left[\tau_{1}<\infty\right]=1$ для любого $z \in B \otimes \mathbf{R}$ и, следовательно, применима лемма 1.

Рассмотрим марковскую цепь $Z^{\prime}=\left(Z_{n}^{\prime}\right)_{n \geqslant 0}$, где $Z_{n}^{\prime}=\left(U_{n}^{\prime}, Y_{n}^{\prime}\right), U_{n}^{\prime}=U_{\tau_{n}}, Y_{n}^{\prime}=$ $Y_{\tau_{n}}, n \geqslant 0$. Если $Z_{0}=z$, то для любого $z \in X$ при $n \geqslant 1$ значения $Z_{n}^{\prime}$ принадлежат $\{e\} \cup D$. Обозначим через $\mathscr{P}^{\prime}$ переходной оператор цепи $Z^{\prime}$. 
Из леммы 1 вытекает следующая ключевая лемма, которая показывает, как изменяется факторизация (16) при «исключении» множества $C=(B \otimes \mathbf{R}) \backslash D$.

Лемма 2. Марковская иепь $Z^{\prime}$ определяется строго субстохастической матрицей $P^{\prime}$ и оператором $\mathscr{L}^{\prime}$, где

$$
\begin{aligned}
P^{\prime} & =\left[I-P F_{d}(C)\right]^{-1} P F_{d}(D), \\
\mathscr{L}^{\prime} f(i, y) & =\left(F\left(i, D_{i}\right)\right)^{-1} \int_{D_{i}} f(i, v) F(i, d v), \quad \text { ecлu } F\left(i, D_{i}\right) \neq 0,
\end{aligned}
$$

а если $F\left(i, D_{i}\right)=0$, то $\mathscr{L}^{\prime} f(i, y)$ можно определить произвольным образом, при этом матрица $F_{d}(A)$ была определена в (3) для любого множества $A$.

Д о к а з а т е л ь с т в о. Здесь вектор-функции, координаты которых равны константам, мы будем обозначать полужирным шрифтом. В этих обозначениях из (15) следует, что $\mathscr{L} \mathbf{f}=\mathbf{f}$. Из (15), (18) и (3) следует, что $\mathscr{L}_{A} \mathbf{f}=F_{d}(A) \mathbf{f}$, отсюда и из (18) вытекает, что $\mathscr{P}_{A} \mathbf{f}=P F_{d}(A) \mathbf{f}$, а значит, $\left(\mathscr{P}_{A}\right)^{l} \mathbf{f}=\left(P F_{d}(A)\right)^{l} \mathbf{f}$ для любого $l \geqslant 1$. Из последнего равенства с $A=C$ и из равенства (18) с $A=D$, учитывая, что $\mathscr{P}_{D} f$ является вектор-функцией, координаты которой равны константам, получаем, что $\left(\mathscr{P}_{C}\right)^{l} \mathscr{P}_{D} f=\left(P F_{d}(C)\right)^{l} P \mathscr{L}_{D} f$. Подставляя это выражение в правую часть первого равенства в (12), получаем, что

$$
\mathscr{P}^{\prime} f=\widetilde{P}^{\prime} \mathscr{L}_{D} f, \quad \text { где } \widetilde{P}^{\prime}=\left[I-P F_{d}(C)\right]^{-1} P .
$$

Нормируя меры, определяющие оператор $\mathscr{L}_{D}$, получаем $(19)$ и $(20)$, при этом если $F\left(i, D_{i}\right)=0$ для некоторого $i$, то соответствующую меру $F^{\prime}(i, \cdot)$ можно выбрать произвольно. Лемма 2 доказана.

Перейдем к доказательству теоремы. Следуя работе [2], мы построим такую последовательность марковских цепей $Z^{k}$ и такую невозрастающую последовательность множеств $D^{k}, k \geqslant 0$, что начальное состояние каждой цепи совпадает с начальным состоянием исходной цепи, а состояние цепи $Z^{k}$ (при $k \geqslant 1$ ) в любой момент $r>0$ совпадает с состояниями исходной цепи в момент $r$-го попадания исходной цепи в множество $D^{k}$. При этом последовательность функций $V^{k}(z), k \geqslant 0$, где $V^{k}(z)$ соответствует выигрышу от остановки при первом попадании цепи $Z^{k}$ в множество $D^{k}$, будет сходиться к функции выигрыша исходной цепи, а последовательность множеств $D^{k}$ будет сходиться к множеству остановки исходной цепи.

Пусть $Z^{0}=Z$ и $\mathscr{P}^{0}=\mathscr{P}$ - переходной оператор цепи $Z^{0}$. Положим $d^{1}=\mathscr{P}^{0} \mathrm{~g}$. Заметим, что $d^{1}$ - это вектор-функция, определенная при всех $z \in B \otimes \mathbf{R}$, координаты которой равны константам. Можно рассматривать $d^{1}$ просто как числовой вектор с соответствующими координатами. Положим $D^{1}=\left\{g(z) \geqslant d^{1}\right\}$. Если $F_{d}\left(D^{1}\right)=I$, то положим $Z^{1}=Z^{0}$.

Если $F_{d}\left(D^{1}\right) \neq I$, то в точках множества $C^{1}=(B \otimes \mathbf{R}) \backslash D^{1}$ останавливаться не имеет смысла, поскольку выигрыш от продолжения на один шаг в этих точках заведомо больше, чем выигрыш от остановки. Поэтому мы «исключим» это множество, рассмотрев цепь $Z^{1}$, которая соответствует цепи $Z^{\prime}$ из леммы 2 с $D=D^{1}$. С цепью $Z^{1}$ поступим точно так же, как с цепью $Z^{0}$, и получим цепь $Z^{2}$, и т.д. В результате получим последовательность цепей $\left(Z^{k}\right)_{k \geqslant 0}, Z_{0}^{k}=Z_{0}^{0}$, соответствующую им последовательность переходных операторов $\left(\mathscr{P}^{k}\right)_{k \geqslant 0}$, последовательность векторов $\left(d^{k}\right)_{k \geqslant 1}$, где

$$
d^{k}=\mathscr{P}^{k-1} g, \quad k \geqslant 1,
$$

и последовательность множеств $\left(D^{k}\right)_{k \geqslant 1}$, где

$$
D^{k}=\left\{z: g(z) \geqslant d^{k}\right\}, \quad k \geqslant 1 .
$$

При $k \geqslant 1$ рассмотрим последовательность вектор-функций

$$
V^{k}(z)=g(z), \quad z \in D^{k}, \quad V^{k}(z)=d^{k}, \quad z \in C^{k}=(B \otimes \mathbf{R}) \backslash D^{k} .
$$


Лемма 3. а) Последовательность $\left(V^{k}(z)\right)_{k \geqslant 1}$ не убьввает и сходится $\kappa$ функиии $V(z)$, которая является ченой игры в задаче оптимальной остановки любой из чепей $Z^{k}, k \geqslant 0$.

б) Последовательность $\left(d^{k}\right)_{k \geqslant 1}$ не убьвает, ограничена и сходится $\kappa$ некоторому $d^{*}$. Последовательность множеств $\left(D^{k}\right)_{k \geqslant 1}$ не возрастает и сходится $\kappa$ такому множеству $D^{*}$, ито $\{e\} \cup D^{*}$ является множеством остановки в задаче оптимальной остановки любой из чепей $Z^{k}, k \geqslant 0$, и иепи $Z^{*}$, соответствующей моментам попадания иепи $Z^{0}$ в множество $D^{*}$. Eсли $d^{l+1}=d^{l}$ для некоторого $l$, то $d^{k}=d^{*}, D^{k}=D^{*}$ nрu $k \geqslant l$.

в) Переходной оператор чепи $Z^{k}$ nри $k \geqslant 1$ и при $k=*$ имеет вид

$$
\mathscr{P}^{k} f=\widetilde{P}^{k} \mathscr{L}_{D^{k}} f, \quad \text { zде } \widetilde{P}^{k}=\left[I-P F_{d}\left(C^{k}\right)\right]^{-1} P,
$$

так что иепь $Z^{k}$ определяется матричей $P^{k}=\widetilde{P}^{k} F\left(D^{k}\right)$ и оператором $\mathscr{L}^{k}$, который в случае существования $\left[F\left(D^{k}\right)\right]^{-1}$ имеет вид $\mathscr{L}^{k}=\left[F\left(D^{k}\right)\right]^{-1} \mathscr{L}_{D^{k}}$, а в общем случае определяется согласно лемме 2.

г) Пусть $d^{*}=\left(d_{1}^{*}, \ldots, d_{m}^{*}\right)$, и пусть $G(i, \cdot), i \in B,-$ распределение величинь $g\left(i, \widehat{Y}_{i}\right)$, где $\widehat{Y}_{i}$ имеет распределение $F(i, \cdot)$. Если существует такое $\varepsilon>0$, ито $G\left(i,\left\{\left(-\varepsilon+d_{i}^{*}, d_{i}^{*}\right)\right\}\right)=0$ для всех $i \in B$, то существует такое $k^{*}$, ито $Z^{s}=Z^{k^{*}}$ nри $s \geqslant k^{*}$.

Д о к а з а т е л ь с т в о. Из (22), (23) и следствия 1 получаем, что

$$
d^{k+1} \geqslant d^{k}, \quad k \geqslant 1 \text {, }
$$

а значит, из (23) следует, что

$$
D^{k+1} \subseteq D^{k}, \quad k \geqslant 1 .
$$

Заметим теперь, что цепь $Z^{k}$ получается из цепи $Z^{k-1}$ «исключением» множества $C^{k}$, т.е. значение $Z^{k}$ в момент $n \geqslant 1$ совпадает со значением $Z^{k-1}$ в момент $n$-го попадания цепи $Z^{k-1}$ в множество $D^{k}$. Из (27) следует, что эта же цепь получится из цепи $Z^{0}$, если сразу «исключить» множество $C^{k}$, а значит, в соответствии с $(21)$

$$
\mathscr{P}^{k} f=\left[I-P F_{d}\left(C^{k}\right)\right]^{-1} P \mathscr{L}_{D^{k}} f .
$$

Из строгой субстохастичности матрицы $P$ следует, что элементы матриц $[I-$ $\left.P F_{d}\left(C^{k}\right)\right]^{-1}$ ограничены. Согласно предположению, сделанному при формулировке модели, $\int_{\mathbf{R}} g(i, v) F(i, d v)$ определен и конечен при каждом $i \in B$. Отсюда и из (28) и $(22)$ следует, что последовательность векторов $\left(d^{k}\right)_{k \geqslant 1}$ ограничена. В силу (26) она сходится к некоторому вектору $d^{*}$. А значит, в силу (23) и (27), последовательность множеств $\left(D^{k}\right)_{k \geqslant 1}$ сходится к некоторому множеству $D^{*}$, и, в силу $(23)$, последовательность матриц $\left(P^{k}\right)_{k \geqslant 1}$ сходится к матрице $P^{*}=\left[I-P F_{d}\left(C^{*}\right)\right]^{-1} P$, где $C^{*}=(B \otimes \mathbf{R}) \backslash D^{*}$. Цепь, отвечающая соответствующему $\mathscr{P}^{*}$, получается исключением $C^{*}$ из любой из цепей $Z^{k}, k \geqslant 0$. Если $d^{l+1}=d^{l}$ для некоторого $l$, то из $(23),(22),(25)$ и доказанной сходимости следует, что $d^{k}=d^{*}, D^{k}=D^{*}$ при $k \geqslant l$.

Заметим теперь, что $V^{k}(z), k \geqslant 1$, есть выигрыш от остановки в момент первого попадания любой из цепей $Z^{s}, 0 \leqslant s \leqslant k$, в множество $D^{k}$. Поэтому $V^{k}(z) \leqslant V(z)$, $k \geqslant 1$. Отсюда и из $(24),(22)$ и $(26)$ следует, что $V^{k}(z)$ сходится к некоторой функции $V^{*}(z)$, для которой $V^{*}(z) \leqslant V(z)$. Из (23) и $(24)$ следует, что $V^{k}(z)=g(z) \geqslant d^{k}=$ $\mathscr{P}^{k-1} g(z)$ при $z \in D^{k}$ и $V^{k}(z)=d^{k}=\mathscr{P}^{k-1} g(z)>g(z)$ при $z \in C^{k}$. Переходя в этих соотношениях к пределу, получаем, что $V^{*}(z)$ удовлетворяет уравнению оптимальности (9). Но, согласно утверждению $1, V(z)$ - минимальное решение уравнения оптимальности. Из $V^{*}(z) \leqslant V(z)$ получаем, что $V^{*}(z)=V(z)$. Утверждения а), б) и в) доказаны.

Докажем пункт г). Из условия этого пункта и из сходимости $d^{k}$ к $d^{*}$ следует, что существует такое $k^{*}$, что $d^{k}>-\varepsilon I+d^{*}$ при всех $k \geqslant k^{*}$. Но тогда $\mathscr{L}_{D^{k}}=\mathscr{L}_{D^{*}}$ при всех $k \geqslant k^{*}$. А значит, $d^{k}=d^{*}, D^{k}=D^{*}$ при всех $k \geqslant k^{*}$. Лемма 3 доказана. 
Утверждение теоремы очевидно следует из утверждений леммы.

Соотношения (22)-(25) дают эффективный алгоритм построения функции выигрыша и множества остановки за конечное число шагов для случая, когда носители распределений всех величин $g\left(i, \widehat{Y}_{i}\right)$ не имеют конечных предельных точек. Продемонстрируем это на следующем примере, заметив предварительно, что при проведении расчетов при переходе от $k$ к $k+1$ не обязательно сразу исключать все множество, где $d^{k} \leqslant g(z)<d^{k+1}$, а можно на каждом шаге исключать только его часть.

$\Pi$ р и м е р. Рассмотрим задачу 3 , в которой множество $B$ состоит из двух элементов. Если цепь $\widetilde{U}$ находится в состоянии 1 , то подбрасывается правильная кость, так что распределение $F(1, \cdot)$ сосредоточено с равными вероятностями в точкаx $1,2,3,4,5,6$, а если цепь $\widetilde{U}$ находится в состоянии 2 , то подбрасывается правильный тетраэдр, так что распределение $F(2, \cdot)$ сосредоточено с равными вероятностями в точках $1,2,3,4$. Задано значение $\beta$ параметра дисконтирования и вероятности $p_{i}$ того, что цепь $\widetilde{U}$ останется в состоянии $i$, так что вероятность перехода из состояния $i$ в состояние $3-i$ равна $q_{i}=1-p_{i}, i=1,2$. Соответствующая цепь $Z$ определяется матрицей $P=\left[\begin{array}{ll}p_{1} \beta, & q_{1} \beta \\ q_{2} \beta, & p_{2} \beta\end{array}\right]=\beta \widetilde{P}$. Рассматривается задача оптимальной остановки с $g(1, y)=g(2, y)=y$.

Для целых чисел $k_{1}, k_{2}, 0 \leqslant k_{1} \leqslant 6,0 \leqslant k_{2} \leqslant 4$, положим $D\left(k_{1}, k_{2}\right)=\{z=(i, j): i=$ $\left.1, k_{1}<j \leqslant 6 ; i=2, k_{2}<j \leqslant 4\right\}$. Для оператора $\mathscr{P}^{\prime}$, соответствующего согласно лемме 2 множеству $D=D\left(k_{1}, k_{2}\right)$, обозначим $d\left(k_{1}, k_{2}\right)=\left[\begin{array}{l}d_{1}\left(k_{1}, k_{2}\right) \\ d_{2}\left(k_{1}, k_{2}\right)\end{array}\right]=\mathscr{P}^{\prime} g$. $\mathrm{B}$ этом случае в (21) $P=\beta\left[\begin{array}{ll}p_{1}, & q_{1} \\ q_{2}, & p_{2}\end{array}\right], F_{d}(C)=\left[\begin{array}{cc}k_{1} / 6, & 0 \\ 0, & k_{2} / 4\end{array}\right], \mathscr{L}_{D} g=\left[\begin{array}{l}3.5-a_{k_{1}} \\ 2.5-a_{k_{2}}\end{array}\right]$, где $a_{k_{1}}=k_{1}\left(k_{1}+1\right) / 12, a_{k_{2}}=k_{2}\left(k_{2}+1\right) / 8$.

Используя явную формулу для обращения фигурирующей в (21) матрицы размера $2 \times 2$ и приводя подобные члены в определителе, получаем

$$
\left[I-P F_{d}(C)\right]^{-1}=\frac{1}{\gamma}\left[\begin{array}{rr}
1-\beta p_{2} k_{2} / 4, & \beta q_{1} k_{2} / 4 \\
\beta q_{2} k_{1} / 6, & 1-\beta p_{1} k_{1} / 6
\end{array}\right],
$$

где $\gamma \equiv \gamma\left(k_{1}, k_{2}\right)=1-\beta\left(p_{1} k_{1} / 6+p_{2} k_{2} / 4\right)+\beta^{2}\left(p_{1}-q_{2}\right) k_{1} k_{2} / 24-$ это определитель матрицы $I-P F_{d}(C)$. Отсюда, используя $(21)$ и определение вектора $d\left(k_{1}, k_{2}\right)$, получаем в результате умножения матриц, что

$$
\begin{aligned}
& d_{1}\left(k_{1}, k_{2}\right)=\frac{\beta}{\gamma}\left(2.5-a_{k_{2}}+p_{1}\left(1+a_{k_{2}}-a_{k_{1}}\right)+\beta\left(q_{2}-p_{1}\right) \frac{k_{2}}{4}\left(3.5-a_{k_{1}}\right)\right), \\
& d_{2}\left(k_{1}, k_{2}\right)=\frac{\beta}{\gamma}\left(2.5-a_{k_{2}}+q_{2}\left(1+a_{k_{2}}-a_{k_{1}}\right)+\beta\left(q_{2}-p_{1}\right) \frac{k_{1}}{6}\left(2.5-a_{k_{2}}\right)\right) .
\end{aligned}
$$

Пусть $[a]$ обозначает целую часть числа $a$. Согласно (22)-(24), имеем: $d^{1} \equiv$ $\left(d_{1}^{1}, d_{2}^{1}\right)=d(0,0), d^{i+1} \equiv\left(d_{1}^{i+1}, d_{2}^{i+1}\right)=d\left(k_{1}^{i}, k_{2}^{i}\right)$, где $k_{1}^{i}=\left[d_{1}^{i}\right], k_{2}^{i}=\left[d_{2}^{i}\right]$. Приведем результат вычислений для некоторых конкретных значений.

\begin{tabular}{|c|c|c|c|c|c|c|c|c|}
\hline № & $\beta$ & $p_{1}$ & $p_{2}$ & $d^{1}$ & $d^{2}$ & $d^{3}$ & $d^{4}$ & $d^{5}$ \\
\hline 1 & 0.7 & 0.60 & 0.30 & $2.17,2.24$ & $2.44,2.50$ & $2.44,2.50$ & & \\
\hline 2 & 0.8 & 0.60 & 0.30 & $2.48,2.56$ & $2.96,3.01$ & $2.957,3.014$ & $2.957,3.014$ & \\
\hline 3 & 0.8 & 0.64 & 0.20 & $2.51,2.64$ & $2.99,3.09$ & $3.005,3.092$ & $3.006,3.093$ & $3.006,3.093$ \\
\hline 4 & 0.9 & 0.80 & 0.05 & $2.97,3.11$ & $3.76,3.81$ & $3.938,4.001$ & $3.938,4.001$ & $3.938,4.001$ \\
\hline
\end{tabular}

Из последнего утверждения пункта б) леммы 3 вытекают следующие результаты. В случае 1 множество остановки находится за один шаг и после подбрасывания как 
кости, так и тетраэдра нужно останавливаться при выпадении числа, большего или равного трем. В случае 2 множество остановки находится за два шага и после подбрасывания кости нужно останавливаться при выпадении числа, большего или равного трем, а после подбрасывания тетраэдра нужно останавливаться только при выпадении четверки. В случае 3 множество остановки находится за три шага и после подбрасывания как кости, так и тетраэдра нужно останавливаться при выпадении числа, большего или равного четырем. В случае 4 множество остановки находится за три шага и после подбрасывания кости нужно останавливаться при выпадении числа, большего или равного четырем, а после подбрасывания тетраэдра останавливаться не нужно.

\section{СПИСОК ЛИТЕРАТУРЫ}

1. Дынкин Е. Б., Юшкевич А. А. Теоремы и задачи о процессах Маркова. М.: Наука, $1967,232 \mathrm{c}$.

2. Пресман Э. Л. Об алгоритме Сонина решения задачи оптимальной остановки. Труды Четвертой международной конференции по проблемам управления (26-30 января 2009 г.). М.: Институт проблем управления им. В. А. Трапезникова РАН, 2009, c. 300-309.

3. Роббинс Г., Сигмунд Д., Чао И. Теория оптимальных правил остановки. М.: Наука, 1977, 168 с.; пер. с англ.: Chow Y.S., Robbins H., Sigmund D. Great Expectations: The Theory of Optimal Stopping. Boston: Houghton Mifflin, 1971, $141 \mathrm{p}$.

4. Ширяев A.H. Статистический последовательный анализ: оптимальные правила остановки. М.: Наука, 1969, 232 с.; 2-е изд.: М.: Наука, 1976, 272 с.

5. Cinlar E. Introduction to Stochastic Processes. Englewood Cliffs: Prentice-Hall, 1975, $402 \mathrm{p}$.

6. Ferguson T.S. Optimal Stopping and Applications. http://www.math.ucla.edu/ - tom/

7. Peskir G., Shiryaev A.N. Optimal Stopping and Free-Boundary Problems. Basel: Birkhäuser, 2006, 500 p.

8. Puterman M. L. Markov Decision Processes: Discrete Stochastic Dynamic Programming. New York: Wiley, 1994, 649 p.

9. Sonin I. M. Two simple theorems in the problems of optimal stopping. - Proceedings of the 8th INFORMS Appl. Probab. Conf., Atlanta, Georgia, 1995, p. 27.

10. Sonin I. M. The elimination algorithm for the problem of optimal stopping. - Math. Methods Oper. Res., 1999, v. 49, № 1, p. 111-123.

11. Sonin I. M. The state reduction and related algorithms and their applications to the study of Markov chains, graph theory, and the optimal stopping problem. - Adv. Math., 1999, v. 145, № 2, p. 159-188.

12. Sonin I. M. The optimal stopping of «seasonal» observations. - Proceedings of the 11th INFORMS Appl. Probab. Conf., New York, 2001, p. 28.

13. Sonin I. M. The optimal stopping of a Markov chain and recursive solution of Poisson and Bellman equations. - From Stochastic Calculus to Mathematical Finance. Berlin: Springer-Verlag, 2006, p. 609-621.

Поступила в редакцию 28.III. 2008

Исправленный вариант

26.III.2009 